\title{
Pharyngo-Cervico-Brachial Variant of Guillain-Barré or Miller Fisher Syndromes? When the Overlap Is Misleading
}

\author{
Leila Tamaoui ${ }^{1, *}$, Mounia Rahmani ${ }^{1}$, Hajar Touati ${ }^{1}$, Leila Errguig ${ }^{2}$, Maria Benabdeljlil ${ }^{1}$ \\ and Saadia Aidi ${ }^{1}$ \\ 1 Department of Neurology A and Neuropsychology, Hôpital des Spécialités, IBN SINA University Hospital, \\ Mohammed 5 University of RABAT, Rabat 10100, Morocco; mouniarahmani4@gmail.com (M.R.); \\ hajar.touati25@gmail.com (H.T.); benab.maria@yahoo.fr (M.B.); aidi_sadia@yahoo.fr (S.A.) \\ 2 Department of Clinical Neurophysiology, Hôpital des Spécialités, IBN SINA University Hospital, \\ Mohammed 5 University of Rabat, Rabat 10100, Morocco; lerrguig@hotmail.com \\ * Correspondence: tamaouileila@gmail.com
}

Received: 1 September 2020; Accepted: 20 November 2020; Published: 24 November 2020

check for updates

\begin{abstract}
Miller Fisher Syndrome (MFS) is a rare variant of Guillain-Barré Syndrome (GBS). It is largely a clinical diagnosis based on the classical features of ataxia, areflexia, and opthalmoplegia. Its clinical evolution is most often favorable. However, other neurological signs and symptoms may also be present. Supportive laboratory studies (positivity of antibodies, CSF albumin-cytological dissociation and nerve conduction studies) are useful especially in uncommon presentations. We report a case of a 74-year-old patient who exhibited dysphonia and difficulty to swallowing previously to the classic triad of ataxia, areflexia, and opthalmoplegia, characteristic of MFS. CSF analysis demonstrates an albumin-cytological dissociation. Anti-GTa1 antibodies were found but anti-GQ1b antibodies were negative. The patient has spontaneously and completely recovered after 6 weeks.
\end{abstract}

Keywords: Miller Fisher Syndrome; Guillain Barré Syndrome variant; dysphonia; dysphagia

\section{Introduction}

Miller Fisher's syndrome (MFS) is a rare variant of Guillain Barré Syndrome (GBS) characterized by the acute onset of ataxia, ophthalmoparesis, and areflexia. The appearance of symptoms following an infection of the respiratory or digestive tract is a common ground for all GBS variants. The diagnosis of MFS is essentially clinical and it is mostly supported by serum positivity for anti-ganglioside antibodies [1]. Others supportive laboratory findings include CSF albumin-cytological dissociation and presence of sensitive abnormalities in nerve conduction studies. Currently, there are no consensus diagnostic criteria [2]. In addition to the classical form, the spectrum of MFS includes incomplete and extensive forms, with additional central clinical features such as the Bickerstaff rhombencephalitis phenotype or peripheral symptomatology suggesting overlap with classical GBS or its other variants [3]. Of the GQ1b-positive subtypes of GBS, the pharyngeal-cervical-brachial (PCB) variant is most commonly associated with oropharyngeal weakness. Patients with the PCB variant of GBS typically present with rapidly progressive oropharyngeal and cervicobrachial weakness associated with areflexia in the upper limbs. 'Incomplete' PCB variants have been described in the literature; for instance, 'acute oropharyngeal palsy' represents a subtype of PCB in which upper limb weakness is absent [4]. Therefore, our patient appears to reflect an overlap syndrome of MFS with incomplete PCB, given the involvement of bulbar cranial nerves responsible of difficulty of swallowing and dysphonia in addition to the MFS triad, which is an extremely rare clinical presentation. 


\section{Case Presentation}

A 74-year-old hypertensive and diabetic man presented to our emergency department for acute onset over 5 days of right ptosis, dysphonia with nasal voice, difficulty swallowing and horizontal diplopia. Few days later, he noted unsteady gait with dizziness sensation with no nausea nor vomiting. No evidence of previous infectious episode was found. At admission, he was fully alert and oriented to person, place and time with an intact memory. The clinical examination revealed a complete bilateral ophthalmoplegia associated with a slightly right predominant ptosis. When asked to walk, a static ataxia that did not increase by closing the eyes was remarkable. Vibration sense was decreased in the four extremities in addition to a diffuse osteotendinous areflexia. Additionally, there was a paresis of the soft palate, abolition of the nauseated reflex, dysphonia with nasal voice and dysphagia of solids and liquids, which indicated the use of nasogastric tube. The remainder of his examination was unremarkable. We realized urgently a brain Magnetic Resonance Imaging (MRI) including restricted diffusion, T2-weighted and fluid-attenuated inversion recovery (FLAIR) sequences with thin slices of the brainstem to exclude central parenchymal lesions. Gadolinium contrast helps to exclude meningeal pathology, especially at the skull base. Cerebrospinal fluid (CSF) analysis showed albumino-cytological dissociation with normal cells and elevated CSF protein at $1 \mathrm{~g} / \mathrm{dl}$, with a normal glucose level. A large electroneuromyographic examination was realized on day 6 including sensory and motor nerve studies, F waves, $\mathrm{H}$ reflex, and repetitive nerve stimulation in the four limbs and in the facial nerve. Surprisingly, the outcome was symmetrically normal. At this level, we assessed a targeted immunological investigation that revealed positive anti-GT1a antibodies and negative Anti-GQ1B antibodies. The standard biological assessment (blood count-platelet count, blood crase, blood ionogram, renal and hepatic functions), thyroid assessment came out normal. Specific viral infections (syphilitis, hepatitis A, B, C, Human Immune Deficiency Virus) were ruled out.

In view of the clinical presentation, we proposed an intravenous immunoglobulins (IVIG) course, but the treatment was not affordable for the patient. Fortunately, the evolution was marked by spontaneous and rapid improvement even if a specific treatment was not used. Complete resolution of symptoms was observed in 6 weeks.

\section{Discussion}

Our patient has presented with unusual initial symptoms, that were the nasal voice and dysphagia, previously to the classic triad of MFS. This situation may be challenging for the clinician and lead to additional investigations to narrow the differential diagnosis, especially in early disease.

According to their clinical features, GBS and its variant MFS can be classified into different subtypes which together form a continuous spectrum of several overlapping syndromes. The involvement of cranial nerves other than the typical oculomotor ones, seems to be more frequent in the forms of MFS-GBS overlap: facial nerve in 30 to $50 \%$, glossopharyngeal (IX), vagus (X), large hypoglossus (XII), responsible for disorders of swallowing and dysphonia, in $13 \%$ of cases $[5,6]$.

The nasal voice related to palate paresis has been rarely described in MFS [7,8]. Moreover, dysphagia was found a less frequent sign $(2 \%)$ in a clinical series of 50 Japanese patients [9].

Bulbar weakness is a prominent feature of the PCB variant of GBS additionally to the cervicobrachial weakness. It can be isolated in an incomplete PCB known as "acute oropharyngeal palsy" [4].

The classic albumino-cytological dissociation of CSF is common and it was a strong supportive feature to the diagnosis in our case [1]. The electromyography and nerve conduction studies (EMG/NCS) have a more limited role in Miller Fisher's syndrome. They are usually normal in the "pure" Miller Fisher's syndrome. In the opposite case, mild abnormal sensory nerve conduction studies and blink reflex are the most often seen abnormalities [2]. Our patient presented with rich clinical signs, however the EMG/NCS did not reveal any abnormality. This result was not expected and increased the doubts about an overlap between a MFS and an incomplete form of PCB variant of GBS. A literature review found that NCS findings in PCB/FS have been rarely and variably reported. Similar to our patient, the initial NCS could not detect any abnormalities but serial electrophysiological studies may be 
valuable [9-14]. The findings in patient with PCB are more consistent with axonal degeneration rather than demyelination [12-14]. However, some authors have shown patterns suggesting reversible axonal conduction failure as an underlying pathology [12,13].

Based on the newest diagnostic guidelines, $\mathrm{PCB}$ is retained by the presence of the clinical features. EMG/NCS, serologic confirmation as well as CSF albuminocytologic dissociation are supportive features, however, are not mandatory for diagnostic [4].

To further support our diagnosis, brain MRI was done and did not show any brainstem abnormalities ruling out an overlap with Bickerstaff rhomboencephalitis or structural lesions that can mimic the patient's presentation. Hence, MRI is the most early important paraclinical investigation. It should be normal in the case of MFS and PCB variant of GBS, although there may be gadolinium enhancement of proximal cervical nerve roots [3,4].

The anti-GQ1b Antibodies spectrum diseases include GBS subtypes, MFS and Bickerstaff S. GQ1b-seronegative is reported in a minority of MFS patients (approximately 10 to 17\%), and may be related to an insufficient sensitivity method of detection or to the pathogenicity of antibodies against gangliosides other than GQ1b [7]. They were ruled out in this case.

The anti GT1a antibodies often coexist with anti GQ1B, due to their cross-reactivity $[1,15,16]$. In our case, the positivity of anti GT1a may be of particular interest as they are commonly detected in pharyngeal-cervical-brachial variant of GBS patients and are associated with bulbar palsy in up to $70 \%$ of GBS patients $[17,18]$. Their presence further supports the overlap hypothesis. In the largest study of 100 patients with PCB, there were 26 PCB/MFS patients with anti-GT1a and -GQ1b antibodies present in $73 \%$ and $81 \%$ of them, respectively [10].

Therefore, taken together, the classic triad of MFS symptoms following the acute bulbar palsy, the positive GT1a titre and the CSF albuminocytologic dissociation allowed for the final diagnosis of MFS with incomplete PCB overlap.

MFS is usually a self-limited disorder with good prognosis and spontaneous recovery generally 2 weeks after onset. Immunotherapy is not required unless there are features of an overlap syndrome with GBS variant or in case of severe Miller Fisher syndrome who develop swallowing and respiratory difficulties despite lack of supporting evidence of benefit [1]. Thus, we expect treatment hasten recovery. In patients with $\mathrm{PCB}$, treatment guidelines follow those of GBS regarding the immunotherapy. However, bulbar and respiratory functions worth more attention with ongoing assessment to guide the respective use of nasogastric feeding and ventilator support [14].

Our patient needed indeed an immunotherapy course to prevent a severe turn out of his condition. Unfortunately, he did not have any medical insurance and its cost was unaffordable. The evolution was rapidly favorable noted as early as day 10 and the complete recovery was observed within 6 weeks of initial symptoms.

\section{Conclusions}

Our observation aims to draw attention to an overlap syndrome of MFS with incomplete PCB, due to symptoms of oropharyngeal palsy in addition to the MFS triad of ataxia, areflexia and ophthalmoplegia and to reflect the misleading possibility in front of such a rich clinical case. Overall, clinical, immunological and neurophysiological studies provide evidence that PCB makes a continuous spectrum with MFS $[4,10,19]$.

Antiganglioside antibody testing has limited value as negative test result does not rule out GBS. Yet, they may be useful in patient with atypical presentation but their results may be delayed and, therefore, should not be relied on for diagnosis [20].

Patients with MFS usually show good recovery even without specific immunotherapy. However, in case of an overlapping syndrome with PCB immunoglobulin therapy or plasmapheresis is highly recommended in order to abort the axonal damage and improve the patient's clinical prognosis $[10,21]$. 
Author Contributions: L.T.: writing the article and designing the study; M.R.: revising the manuscript; H.T.: receiving and examining the patient; L.E.: performing the ENMG; M.B.: revising the manuscript; S.A.: supervising and directing the study form of the article. All authors have read and agreed to the published version of the manuscript.

Funding: The author(s) received no financial support for the research, authorship, and/or publication of this article.

Conflicts of Interest: On behalf of all authors, the corresponding author states that there is no conflict of interest.

\section{References}

1. Rocha Cabrero, F.; Morrison, E.H. Miller Fisher Syndrome. In StatPearls [Internet]; StatPearls Publishing: Treasure Island, FL, USA, 2019. Available online: https://www.ncbi.nlm.nih.gov/books/NBK507717 (accessed on 26 March 2019).

2. Teener, J.W. Miller Fisher's Syndrome. Semin Neurol. 2012, 32, 512-516. [CrossRef] [PubMed]

3. Wakerley, B.R.; Yuki, N. Mimics and chameleons in Guillain-Barré and Miller Fisher syndromes. Pr. Neurol. 2015, 15, 90-99. [CrossRef] [PubMed]

4. Wakerley, B.R.; Yuki, N. Pharyngeal-cervical-brachial variant of Guillain-Barré syndrome. J. Neurol. Neurosurg. Psychiatry 2014, 85, 339-344. [CrossRef] [PubMed]

5. Wakerley, B.R.; the GBS Classification Group; Uncini, A.; Yuki, N. Guillain-Barré and Miller Fisher syndromes-new diagnostic classification. Nat. Rev. Neurol. 2014, 10, 537-544. [CrossRef] [PubMed]

6. Labodi, L.D.; Adrien, M.; Anne, A.; Muhammad, K.; René, D.; Bernard, B. Miller-fisher syndrome on the borders of guillain-barre syndrome: About one case and review of literature. Clin. Med Rep. 2017, 1, 1-5.

7. Howell, R.J.; Davolos, A.G.; Clary, M.S.; Frake, P.C.; Joshi, A.S.; Chaboki, H. Miller fisher syndrome presents as an acute voice change to hypernasal speech. Laryngoscope 2010, 120, 978-980. [CrossRef] [PubMed]

8. Robbins, M.S.; Roth, S.; Swerdlow, M.L.; Bieri, P.; Herskovitz, S. Optic neuritis and palatal dysarthria as presenting features of post-infectious GQ1b antibody syndrome. Clin. Neurol. Neurosurg. 2009, 111, 465-466. [CrossRef] [PubMed]

9. Mori, M.; Kuwabara, S.; Fukutake, T.; Yuki, N.; Hattori, T. Clinical features and prognosis of Miller Fisher syndrome. Neurology 2001, 56, 1104-1106. [CrossRef] [PubMed]

10. Nagashima, T.; Koga, M.; Odaka, M.; Hirata, K.; Yuki, N. Continuous Spectrum of Pharyngeal-Cervical-Brachial Variant of Guillain-Barré Syndrome. Arch. Neurol. 2007, 64, 1519-1523. [CrossRef] [PubMed]

11. Ropper, A.H. Further Regional Variants of Acute Immune Polyneuropathy. Arch. Neurol. 1994, 51, 671-675. [CrossRef] [PubMed]

12. Rajabally, Y.A.; Hassan-Smith, G.; Notturno, F.; Eames, P.J.; Hayton, T.; Capasso, M.; Uncini, A. Motor and sensory conduction failure in overlap of Guillain-Barré and Miller Fisher syndrome: Two simultaneous cases. J. Neurol. Sci. 2011, 303, 35-38. [CrossRef]

13. Capasso, M.; Notturno, F.; Manzoli, C.; Yuki, N.; Uncini, A. Reversible conduction failure in pharyngeal-cervical-brachial variant of guillain-barré syndrome. Muscle Nerve 2010, 42, 608-612. [CrossRef]

14. Aranyi, Z.; Kovacs, T.; Sipos, I.; Bereczki, D. Miller Fisher syndrome: Brief overview and update with a focus on electrophysiological findings. Eur. J. Neurol. 2012, 19, 15-20. [CrossRef]

15. Ito, M.; Kuwabara, S.; Odaka, M.; Misawa, S.; Koga, M.; Hirata, K.; Yuki, N. Bickerstaff's brainstem encephalitis and Fisher syndrome form a continuous spectrum. J. Neurol. 2008, 255, 674-682. [CrossRef]

16. Ramakrishna, K.N.; Tambe, V.; Kattamanchi, A.; Dhamoon, A.S. Miller Fisher syndrome with bilateral vocal cord paralysis: A case report. J. Med. Case Rep. 2020, 14, 31. [CrossRef]

17. Kim, J.K.; Kim, B.-J.; Shin, H.Y.; Shin, K.J.; Nam, T.-S.; Oh, J.; Suh, B.C.; Yoon, B.-A.; Park, H.T.; Huh, S.-Y.; et al. Acute bulbar palsy as a variant of Guillain-Barré syndrome. Neurology 2015, 86, 742-747. [CrossRef]

18. Noureldine, M.H.A.; Sweid, A.; Ahdab, R. Miller Fisher syndrome presenting as palate paralysis. J. Neuroimmunol. 2016, 298, 130-131. [CrossRef]

19. Chan, Y.-C.; Ahmad, A.; Paliwal, P.; Yuki, N. Non-demyelinating, reversible conduction failure in a case of pharyngeal-cervical-brachial weakness overlapped by Fisher syndrome. J. Neurol. Sci. 2012, 321, $103-106$. [CrossRef] [PubMed] 
20. Leonhard, S.E.; Mandarakas, M.R.; Gondim, F.A.A.; Bateman, K.; Ferreira, M.L.B.; Cornblath, D.R.; Van Doorn, P.A.; Dourado, M.E.; Hughes, R.A.C.; Islam, B.; et al. Diagnosis and management of Guillain-Barré syndrome in ten steps. Nat. Rev. Neurol. 2019, 15, 671-683. [CrossRef]

21. Mori, M.; Kuwabara, S. Fisher syndrome. Curr. Treat Options Neurol. 2011, 13, 71-78. [CrossRef]

Publisher's Note: MDPI stays neutral with regard to jurisdictional claims in published maps and institutional affiliations.

(C) 2020 by the authors. Licensee MDPI, Basel, Switzerland. This article is an open access article distributed under the terms and conditions of the Creative Commons Attribution (CC BY) license (http://creativecommons.org/licenses/by/4.0/). 